\title{
Ultrasound beyond regional anesthesia: formal training?
}

\author{
Ban C. H. Tsui, MD • Vivian Hui Yun Ip, MBChB
}

Published online: 24 March 2011

(C) Canadian Anesthesiologists' Society 2011

In this issue of the Journal, Dugani et al. ${ }^{1}$ present a case concerning a patient undergoing a caudal block for chronic pain treatment wherein a bowel hernia, inferoposterior to the coccygeal ligament, was found incidentally using fluoroscopy and confirmed with ultrasound. The initial procedure was abandoned, and subsequently, the patient underwent coccygeal hernia repair. In this case, Dugani et al. ${ }^{1}$ raise two important issues in both chronic pain management and ultrasound-guided regional anesthesia, namely, "incidental detection" and "the capability of ultrasound to identify nonneural pathology", that are more notable than the authors' ability to define the pathological condition and modify patient management using ultrasound and fluoroscopy. Undoubtedly, the continuing popularity of ultrasound techniques in regional anesthesia will result in the exposure of anesthesiologists to these issues; in fact, an increasing number of such cases are reported in many journals. However, when it comes to diagnosing pathology using ultrasound, most anesthesiologists lack training and experience. Collaboration with radiologists and formal training in ultrasound are considerations for equipping anesthesiologists to take advantage of the possibilities offered by imaging technologies.

In chronic pain management, pain syndromes can be complex and difficult to diagnose. Coccydynia is no exception, presenting with symptoms from discomfort to acute and severe pain, varying between patients, and changing over time. It can occur after falls, childbirth, repetitive strain, or prior surgery. In many cases, the origin

B. C. H. Tsui, MD (ه) • V. H. Y. Ip, MBChB Department of Anesthesiology and Pain Medicine, University of Alberta Hospital, 8-120 Clinical Sciences Building, Edmonton, AB T6G 2G3, Canada

e-mail: btsui@ualberta.ca of the pain is unclear and the cause unknown. Although a diagnosis of coccydynia often is based solely on the symptoms, other organic causes (e.g., local rashes from shingles) and bone and tissue disorders (fracture) must be ruled out by performing a physical examination, diagnostic imaging, and other appropriate tests.

In many cases, this "incidental detection" can be avoided from the outset; however, in this case, could investigations have been performed prior to the caudal injection to prevent the "on-table" incidental finding? Recently, the American College of Physicians and the American Pain Society proposed a joint clinical practice guideline suggesting a focused history and examination is important to identify the nature of the pain and the warning signs for underlying pathologies. ${ }^{2}$ Appropriate diagnostic imaging should be considered and reserved for patients with severe or progressive neurologic deficits or in cases where serious underlying conditions are suspected. ${ }^{2}$ In the case of Dugani et al., ${ }^{1}$ bowel herniation post-coccygectomy is extremely rare, and the symptoms persisting after surgery would not necessarily prompt further investigations prior to intervention, barring a change of symptoms. This case reminds clinicians to conduct appropriate investigations in patients with nonspecific pain prior to committing to an invasive intervention, especially after a surgical procedure. Nevertheless, despite the most thorough history and examination, diagnoses will occasionally be missed, thus opening the window for "incidental detection" in cases where extensive investigations are not indicated.

Ultrasound is a challenging imaging modality because it is highly operator-dependent in terms of both acquisition and interpretation of the image. The inherent user-dependent sensitivity and specificity of ultrasound could result in either a false negative (missed diagnosis) or a false positive (misinterpretation of an insignificant lesion). Either of 
these results can potentially lead to morbidity for the patient and cost to the health system. As stated by the World Health Organization Scientific Group, "More important than the equipment is the availability of skills. An error in diagnosis because of inadequate education and experience is as dangerous as being without the equipment." ${ }^{3}$ The relevance of the operator's skills to patient care raises the issue of the need for stringent training. In Canada, radiologists undergo three to six months of ultrasound training during their residency, with the option to extend this time with further training in musculoskeletal radiology for neural imaging. The complexity of ultrasound use as a diagnostic device is implicit.

Issues regarding proper ultrasound training are not unique to anesthesiologists; in fact, the radical change of guidelines granting ultrasound privileges to non-radiologists was instigated by emergency medicine physicians. In 1999, the American Medical Association House of Delegates Resolution 802 affirmed that ultrasound use is within the scope of practice of all appropriately trained physicians. ${ }^{4}$ Since then, the American College of Emergency Physicians, the College of Emergency Medicine in the United Kingdom, and the Australasian College for Emergency Medicine have laid out guidelines, competencies, and structured ultrasound training to incorporate within their residency programs. The credentialing process encompasses theoretical and practical training, documentation, assessments, and maintenance of skills; however, the trainers are not necessarily radiologists and teaching is not always modular. Nowadays, emergency physicians who frequently perform focused abdominal sonography for trauma are considered competent in this area. ${ }^{5}$

With respect to transesophageal echocardiography, training for cardiac anesthesiologists is even more structured and the credentialing process is stringent. The Society of Cardiac Anesthesiologists and the American Society of Echocardiography have defined comprehensive competencies for the level of a board certified cardiologist. ${ }^{6}$ Training and credentialing for cardiac anesthesiologists is similar in the United Kingdom, taking place in a cardiothoracic centre during the fellowship year, with three to six months dedicated for transesophageal echocardiography. Skills are taught by cardiac anesthesiologists, often under the supervision of cardiologist colleagues, and are supplemented by courses and workshops. However, anesthesia residents have limited exposure to and experience with echocardiography in their cardiac module where ad hoc teaching occurs but is restricted by the demands on the trainers and trainees during an intense cardiac case. Unless provisions are made during the residency program, there will be little opportunity to learn the requisite skills.

The same applies to transthoracic echocardiography, a useful noninvasive diagnostic and monitoring tool that has the potential to have a positive impact on patient management, especially in intensive care and perioperative settings. Guidelines have been established recently by the WINFOCUS (World Interactive Network Focused on Critical UltraSound) ECHO-ICU Group to aid structured training and credentialing in intensive care to those interested in developing the imaging skills required of intensivists. ${ }^{6}$ The structure of training is similar to that in emergency medicine and uses a continual opportunistic approach under an accredited trainer.

Currently, there is no radiology module during Canadian anesthesia residency training, leaving new practitioners with limited and inconsistent exposure to imaging techniques and interpretation. This is unfortunate; familiarity with ultrasound and sonoanatomy helps to establish a solid foundation for ultrasound-guided regional anesthesia and for the identification of sonopathologies that may improve management in perioperative and intensive care settings, as Dugani et al. ${ }^{1}$ illustrate.

A formal rotation in radiology of at least one month would greatly facilitate the basic practical skills of Canadian anesthesia residents in ultrasound, sonoanatomy, echocardiography, and interpretation of radiographs. In fact, many teaching hospitals have adopted a similar approach for residents in obstetrics/gynecology, orthopedics, neurosurgery, and emergency medicine. Multidisciplinary team meetings with radiologists are a routine part of training in various medical and surgical residency programs in the UK. Incorporating radiology training into the anesthesia residency program will equip future practitioners with the knowledge and skills in radiology that are increasingly essential to our field. It is essential to have a structured curriculum that is targeted to the requirements of anesthesiologists and includes clearly defined objectives and collaboration with the radiology department.

Sites et al. ${ }^{7}$ discuss the obligation of the operator to acquire the knowledge of both normal anatomy and sonopathology in an ultrasound examination, an expansion of the traditional role of the anesthesiologist. While the short duration would necessarily limit the knowledge and skills acquired during a training module, residents should acquire an understanding of the basics as well as an appreciation of the need to collaborate with radiologists and seek expert opinion in situations beyond their experience.

Recognizing the challenges of ultrasound-guided regional anesthesia training, the American Society of Regional Anesthesia and Pain Medicine (ASRA) and the European Society of Regional Anaesthesia and Pain Therapy Joint Committee have recently published guidelines and recommendations for the core competencies and training pathways. ${ }^{8}$ With such guidance, it is our view that many regional anesthesiologists will become familiar with normal nerve sonographic anatomy and ultrasound 
approaches to regional blocks; however, one must be aware that there are fundamental differences between ultrasound use to improve a relatively blind landmark-based procedure (i.e., regional block) and ultrasound use for diagnostic purposes.

Although Dugani et al. ${ }^{1}$ describe a case in which incidental diagnosis using fluoroscopy and ultrasound had a positive impact on patient management, this expansion to a broad spectrum of diagnostic pathology lies clearly outside the training and experience of most anesthesiologists. Given this challenge, there are three routes to take: the first approach is to prevent incidental diagnosis where possible by obtaining a thorough history and performing physical examination and investigations. The second approach is to assess the need for an anesthesia residency program that incorporates and introduces a formal imaging rotation in radiology to solidify the foundation of future anesthesiologists in ultrasound knowledge and training. The third approach is to collaborate with our radiology colleagues and send out an early "call for help" once we are faced with a situation outside our expertise, just as we would follow the American Society of Anesthesiologists' recommendation to "call for help" when facing a "cannot intubate, cannot ventilate" situation in airway management. As the use of ultrasound continues to grow and evolve, it is our view that it will become incorporated into the patient management role of the anesthesiologist much as have other diagnostic tools, such as the stethoscope and the electrocardiogram. We look forward to the initiation of constructive dialogue within our anesthesiology community regarding the best means to equip future anesthesiologists with the ultrasound skills required to optimize patient care.

\section{L'échographie au-delà de l'anesthésie régionale : une formation structurée?}

Dans ce numéro du Journal, Dugani et coll. ${ }^{1}$ présentent le cas d'une patiente recevant un bloc caudal pour traiter une douleur chronique et chez qui on a découvert par hasard une hernie intestinale inférieure et postérieure au ligament coccygien à l'aide de la fluoroscopie, et confirmée par échographie. L'intervention prévue a été interrompue, et la patiente a par la suite subi une herniorraphie coccygienne. Dans ce cas, Dugani et coll. ${ }^{1}$ soulèvent deux points importants qui touchent autant à la prise en charge de la douleur chronique qu'à l'anesthésie régionale par échoguidage, soit «la découverte fortuite » et la «capacité de l'échographie à identifier des pathologies non neurales »-deux éléments qu'il convient de souligner encore davantage que la capacité des auteurs à déterminer la condition pathologique de la patiente et modifier sa prise en charge à l'aide de l'échographie et de la fluoroscopie. Il ne fait aucun doute que les anesthésiologistes seront de plus en plus exposés à de tels problèmes, étant donné la popularité constante des techniques d'échographie en anesthésie régionale; en fait, un nombre croissant de cas semblables sont rapportés dans bon nombre de revues. Cependant, quand il est question de poser un diagnostic de pathologie à l'aide de l'échographie, la formation et l'expérience font toutes deux défaut à la plupart des anesthésiologistes. La collaboration avec les radiologistes et une formation structurée en échographie font partie des options à envisager pour mieux préparer les anesthésiologistes à tirer pleinement parti du potentiel des technologies d'imagerie.

Dans le domaine de la prise en charge de la douleur chronique, les syndromes douloureux peuvent s'avérer complexes et difficiles à diagnostiquer. La coccygodynie ne fait pas exception; cette pathologie s'accompagne de symptômes allant d'un inconfort à des douleurs aiguës et graves, varie d'un patient à l'autre, et évolue au fil du temps. Elle peut se manifester à la suite d'une chute, d'un accouchement, d'un effort répété, ou d'une chirurgie antérieure. Dans bon nombre de cas, l'origine de la douleur est peu claire et sa cause inconnue. Bien que souvent, un diagnostic de coccygodynie se fonde exclusivement sur des symptômes, les autres causes organiques (par ex. des éruptions cutanées locales d'herpès) et des troubles au niveau des os et des tissus (fractures) doivent être écartées en réalisant un examen physique, un diagnostic par imagerie et d'autres tests adéquats.

Dans de nombreux cas, cette «découverte fortuite» peut être évitée d'entrée de jeu; toutefois, dans le cas présenté ici, d'autres examens auraient-ils pu être réalisés avant l'injection caudale afin d'éviter cette découverte fortuite "sur la table d'opération »? L'American College of Physicians et l'American Pain Society ont récemment proposé un guide conjoint de pratique clinique soulignant l'importance d'un examen ciblé et d'une analyse des antécédents afin de déterminer la nature de la douleur et les signes avant-coureurs de pathologies sous-jacentes. ${ }^{2}$ Une imagerie diagnostique adaptée devrait être envisagée et réservée aux patients souffrant de troubles neurologiques graves ou évolutifs ou dans les cas où des conditions sous-jacentes graves sont soupçonnées. ${ }^{2}$ Dans le cas présenté par Dugani et coll., ${ }^{1}$ une hernie intestinale post-coccygectomie est extrêmement rare, et les symptômes persistant après la chirurgie n'inciteraient pas forcément à la réalisation d'autres examens plus poussés avant une intervention, sauf en cas d'évolution de ces symptômes. Ce cas rappelle aux cliniciens qu'ils doivent réaliser des tests adaptés chez les patients souffrant de 
douleurs non spécifiques avant d'initier une procédure effractive et ce, particulièrement après une intervention chirurgicale. Néanmoins, malgré les examens les plus complets et la révision des antécédents, il est possible qu'occasionnellement des diagnostics passent inaperçus, ouvrant dès lors la porte aux « découvertes fortuites » dans les cas où des examens approfondis ne sont pas indiqués.

L'échographie est une modalité d'imagerie difficile parce qu'elle dépend beaucoup de l'opérateur, tant en termes d'acquisition que d'interprétation des images. La sensibilité et la spécificité dépendantes de l'utilisateur inhérentes à l'échographie pourraient résulter en un faux négatif (diagnostic manqué) ou un faux positif (mauvaise interprétation d'une lésion peu importante). L'un ou l'autre résultat peut potentiellement entraîner une morbidité pour le patient et des coûts pour le système de santé. Comme le Groupe scientifique de l'Organisation mondiale de la Santé le déclare, "La disponibilité de compétences est plus importante encore que celle de matériel. Une erreur de diagnostic en raison d'une formation inadaptée et du manque d'expérience est tout aussi dangereuse qu'un manque de matériel. $»^{3}$ La pertinence des compétences de l'opérateur en ce qui touche aux soins des patients soulève la question de la nécessité d'une formation rigoureuse. Au Canada, les radiologistes suivent trois à six mois de formation en échographie au cours de leur résidence, et peuvent, s'ils le souhaitent, prolonger cette période en suivant une formation approfondie en radiologie musculosquelettique pour l'imagerie neuronale. La complexité d'utilisation de l'échographie en tant qu' appareil de diagnostic est implicite dans la durée de cette formation.

Les questions liées à une formation adéquate en échographie ne sont pas uniques à l'anesthésiologie; en effet, le changement radical des directives accordant des privilèges d'échographie aux non-radiologistes s'est fait à l'instigation des urgentologues. En 1999, la Résolution 802 de la Chambre des délégués de l'American Medical Association déclarait que l'utilisation de l'échographie relevait du domaine de pratique de tous les médecins adéquatement formés. ${ }^{4}$ Depuis, l'American College of Emergency Physicians, le College of Emergency Medicine au Royaume-Uni et l'Australasian College for Emergency Medicine ont émis des directives ainsi qu'une liste de qualifications et mis en place une formation structurée en échographie qui sera intégrée à leurs programmes de résidence. Le processus de certification couvre une formation théorique et pratique, de la documentation, des évaluations et un maintien des compétences; cependant, les formateurs ne sont pas nécessairement des radiologistes et l'enseignement ne se fait pas toujours de façon modulaire. De nos jours, les urgentologues qui réalisent fréquemment des échographies abdominales ciblées pour détecter les traumatismes sont considérés comme étant compétents dans ce domaine. ${ }^{5}$

En ce qui concerne l'échocardiographie transœsophagienne, la formation des anesthésiologistes cardiaques est encore plus structurée et le processus de certification rigoureux. La Society of Cardiac Anesthesiologists et l'American Society of Echocardiography ont défini un cadre de compétences exhaustif pour le niveau d'un cardiologue détenteur d'un certificat de spécialiste. ${ }^{6}$ La formation et la certification des anesthésiologistes cardiaques est similaire au Royaume-Uni et se déroule dans un centre cardiothoracique pendant l'année de stage (le fellowship), dont trois à six mois sont consacrés à l'échographie transœsophagienne. Les compétences sont enseignées par des anesthésiologistes cardiaques, souvent sous la supervision de collègues cardiologues, auxquelles s'ajoutent cours et ateliers. Toutefois, les résidents en anesthésie ne sont que peu exposés et possèdent peu d'expérience en matière d'échocardiographie dans leur module cardiaque, où l'enseignement $a d$ hoc a lieu mais est limité par les contraintes imposées aux formateurs et aux stagiaires pendant un cas cardiaque intense. Sauf si des dispositions sont prises pendant le programme de résidence, les anesthésiologistes en formation n'auront que peu d'occasions d'acquérir les compétences requises.

Ceci s'applique également à l'échocardiographie transthoracique, un outil diagnostique et de monitorage non effractif utile qui peut potentiellement avoir un impact positif sur la prise en charge des patients, particulièrement en soins intensifs et dans le cadre périopératoire. Des directives ont récemment été établies par le groupe WINFOCUS (World Interactive Network Focused on Critical UltraSound) ECHO-ICU afin de soutenir les formations structurées et la certification en soins intensifs des personnes intéressées à acquérir les compétences d'imagerie nécessaires aux intensivistes. ${ }^{6} \mathrm{La}$ structure de la formation ressemble à celle en médecine d'urgence et se fonde sur une approche opportuniste continue sous un formateur certifié.

À l'heure actuelle, il n'existe pas de module de radiologie pendant la résidence en anesthésie au Canada; par conséquent, les nouveaux praticiens ne profitent que d'une exposition limitée et inadéquate aux techniques d'imagerie et à l'interprétation des images. Ceci est fâcheux; une bonne connaissance de l'échographie et de la sonoanatomie contribue à établir des bases solides pour l'anesthésie régionale par échoguidage et l'identification des pathologies par échographie, ce qui pourrait améliorer la prise en charge des patients dans les cadres périopératoires et de soins intensifs, comme Dugani et coll. ${ }^{1}$ le démontrent.

Un module de formation structuré en radiologie d'une durée minimale d'un mois faciliterait grandement l'acquisition des compétences pratiques de base pour les résidents canadiens en anesthésie en échographie, en 
sonoanatomie, en échocardiographie, et en interprétation des radiographies. En fait, de nombreux hôpitaux universitaires ont adopté une approche semblable pour les résidents en gynécologie/obstétrique, en orthopédie, en neurochirurgie, et en médecine d'urgence. Des réunions pluridisciplinaires avec des radiologistes font partie intégrante de la formation dans plusieurs programmes de résidence en médecine et en chirurgie au Royaume-Uni. L'intégration d'un module de radiologie dans le programme de résidence en anesthésie munira les futurs praticiens des connaissances et des compétences en radiologie qui sont de plus en plus cruciales dans notre domaine. Il est essentiel d'avoir un programme de cours structuré et ciblé pour répondre aux exigences des anesthésiologistes, un programme qui comprenne des objectifs clairement définis et favorise une collaboration avec le département de radiologie.

Sites et coll. ${ }^{7}$ discutent de l'obligation qui incombe à l'opérateur d'acquérir les connaissances de l'anatomie normale et de la sonopathologie lors d'un examen échographique, ce qui constitue une expansion du rôle traditionnel de l'anesthésiologiste. Alors que la courte durée d'un module de formation limiterait nécessairement les connaissances et les compétences acquises, les résidents devraient être à même de comprendre les bases tout en appréciant le besoin de collaboration avec les radiologistes et de demander une opinion d'expert lors de situations allant au-delà de leur expérience.

Conscient des défis posés par la formation en anesthésie régionale par échoguidage, le comité conjoint de l' American Society of Regional Anesthesia and Pain Medicine (ASRA) et de l'European Society of Regional Anaesthesia and Pain Therapy a récemment publié des directives et des recommandations concernant les compétences clés et les possibilités de formation. ${ }^{8}$ Selon nous, avec de tels outils, plusieurs anesthésiologistes intéressés en anesthésie loco-régionale vont se familiariser avec l'anatomie nerveuse normale par sonographie et les approches échographiques aux blocs régionaux; cependant, il faut garder à l'esprit qu'il existe des différences fondamentales entre l'utilisation de l'échographie pour améliorer une intervention fondée sur des repères plus ou moins en aveugle (c.-à-d. des blocs loco-régionaux) et l'utilisation de l'échographie à des fins diagnostiques.

Bien que Dugani et coll. ${ }^{1}$ décrivent un cas dans lequel le diagnostic fortuit établi par fluoroscopie et échographie a eu un impact positif sur la prise en charge de la patiente, l'expansion de cette application pour le diagnostic d'une vaste gamme de pathologies est clairement au-delà de la formation et de l'expérience de bon nombre d' anesthésiologistes. Étant donné le défi existant, trois options se présentent : la première approche est de prévenir les diagnostics fortuits lorsque cela est possible en obtenant des antécédents approfondis et en réalisant un examen physique et des tests. La deuxième approche consiste à évaluer l'utilité d'un programme de résidence en anesthésie qui intègre et introduit un module structuré d'imagerie en radiologie afin de solidifier les bases des anesthésiologistes en formation en matière de connaissances et de formation en échographie. La troisième approche consiste à collaborer avec nos collègues de radiologie et à émettre un « appel à l'aide» précoce aussitôt que nous sommes confrontés à une situation hors de notre champ d'expertise, tout comme nous suivrions la recommandation de l'American Society of Anesthesiologists d'appeler à l'aide lors d'une situation « cannot intubate, cannot ventilate » dans la prise en charge des voies aériennes. Au fur et à mesure que l'utilisation de l'échographie continue d'évoluer et de se répandre, nous pensons que cette modalité sera intégrée dans le rôle de prise en charge du patient par l'anesthésiologiste, tout comme ce fut le cas pour d'autres outils diagnostiques, notamment le stéthoscope et l'électrocardiogramme. Nous nous réjouissons de l'ouverture d'un dialogue constructif dans notre communauté d'anesthésiologie quant à la meilleure façon d'offrir aux anesthésiologistes en devenir les compétences en échographie requises pour optimiser les soins aux patients.

Conflicts of interest None declared.

\section{References}

1. Dugani $S$, Tran DQ, Finlayson RJ. Incidental detection of bowel herniation with ultrasonography and fluoroscopy during a caudal block. Can J Anesth 2011; 58. DOI:10.1007/s12630-011-9489-3.

2. Chou R, Qaseem A, Snow V, et al. Diagnosis and treatment of low back pain: a joint clinical practice guideline from the American College of Physicians and the American Pain Society. Ann Intern Med 2007; 147: 478-91.

3. World Health Organization. WHO technical report series. Training in diagnostic ultrasound: essentials, principles and standards. Geneva; 1998, p. 3. http://whqlibdoc.who.int/trs/WHO_TRS_875. pdf. Accessed February 2011.

4. American Medical Association House of Delegates. Privileging for ultrasound imaging. Resolution 802, December 1999; reaffirmed; Sub. Res 108, June 2000. H-230.960. http://www.ama-assn.org. Accessed January 2011.

5. Ma OJ, Mateer JR, Ogata M, Kefer MP, Wittmann D, Aprahamian $C$. Prospective analysis of a rapid trauma ultrasound examination performed by emergency physicians. J Trauma 1995; 38: 879-85.

6. Price $S$, Via $G$, Sloth $E$, et al. Echocardiography practice, training, accreditation in the intensive care: document for the World Interactive Network Focused on Critical Ultrasound (WINFOCUS). Cardiovasc Ultrasound 2008; 6: 49.

7. Sites BD, Spence BC, Gallagher JD, Beach ML. On the edge of the ultrasound screen: regional anesthesiologists diagnosing nonneural pathology. Reg Anesth Pain Med 2006; 31: 555-62.

8. Sites BD, Chan VW, Neal JM, et al. The American Society of Regional Anesthesia and Pain Medicine and the European Society of Regional Anaesthesia and Pain Therapy joint committee recommendations for education and training in ultrasound-guided regional anesthesia. Reg Anesth Pain Med 2010; 35(2 Suppl): S74-80. 